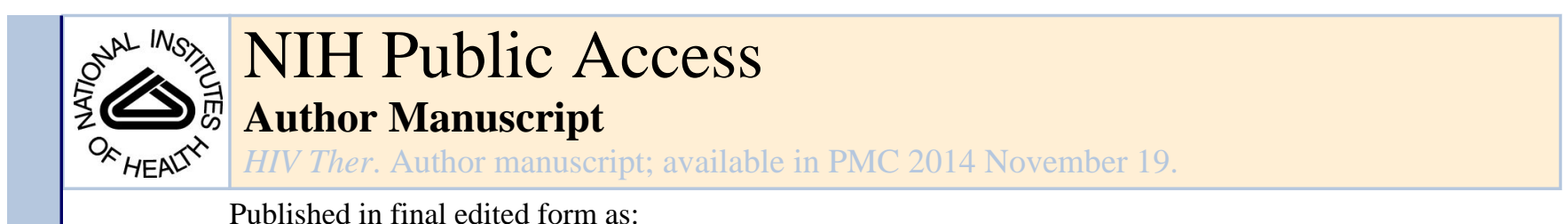

Published in final edited form as:

HIV Ther. 2010 ; 4(6): 703-722. doi:10.2217/hiv.10.52.

\title{
Implementation of cervical cancer prevention services for HIV- infected women in Zambia: measuring program effectiveness
} \author{
Jeffrey SA Stringer ${ }^{1,2}$, and Benjamin $\mathrm{H} \mathrm{Chi}^{1,2}$ \\ ${ }^{1}$ University of Alabama at Birmingham, AL, USA \\ ${ }^{2}$ Centre for Infectious Disease Research in Zambia, Lusaka, Zambia \\ 3University Teaching Hospital, Lusaka, Zambia \\ ${ }^{4}$ Vanderbilt University, TN, USA \\ ${ }^{5}$ National Cancer Institute, MD, USA \\ ${ }^{6}$ University of Michigan, MI, USA \\ ${ }^{7}$ University of Cincinnati, $\mathrm{OH}$, USA \\ ${ }^{8}$ Michigan Cancer Institute, MI, USA
}

Groesbeck P Parham ${ }^{\dagger, 1,2,3}$, Mulindi H Mwanahamuntu ${ }^{2,3}$, Vikrant V Sahasrabuddhe ${ }^{4,5}$, Andrew O Westfall ${ }^{2}$, Kristin E King ${ }^{6}$, Carla Chibwesha ${ }^{1,2}$, Krista S Pfaendler ${ }^{7}$, Gracilia Mkumba $^{2,3}$, Victor Mudenda ${ }^{3}$, Sharon Kapambwe ${ }^{2,3}$, Sten H Vermund ${ }^{4}$, Michael L Hicks ${ }^{8}$,

\begin{abstract}
Background-Cervical cancer kills more women in low-income nations than any other malignancy. A variety of research and demonstration efforts have proven the efficacy and effectiveness of low-cost cervical cancer prevention methods but none in routine program implementation settings of the developing world, particularly in HIV-infected women.
\end{abstract}

Methods-In our public sector cervical cancer prevention program in Zambia, nurses conduct screening using visual inspection with acetic acid aided by digital cervicography. Women with visible lesions are offered same-visit cryotherapy or referred for histologic evaluation and clinical management. We analyzed clinical outcomes and modeled program effectiveness among HIVinfected women by estimating the total number of cervical cancer deaths prevented through screening and treatment.

Results-Between 2006 and 2008, 6572 HIV-infected women were screened, 53.6\% (3523) had visible lesions, 58.5\% (2062) were eligible for cryotherapy and 41.5\% (1461) were referred for histologic evaluation. A total of $75 \%$ (1095 out of 1462) of patients who were referred for evaluation complied. Pathology results from 65\% (715 out of 1095) of women revealed benign abnormalities in $21 \%$ (151), cervical intraepithelial neoplasia (CIN) I in 30\% (214), CIN 2/3 in $33 \%$ (235) and invasive cervical cancer in $16.1 \%$ (115, of which $69 \%$ were early stage). Using a

(C) 2010 Future Medicine Ltd

${ }^{\dagger}$ Author for correspondence: University of Alabama at Birmingham, Centre for Infectious Disease Research in Zambia, Plot \# 5977 Benekale Road, Northmead, Lusaka, Zambia, Tel.: +260 978124 392, Fax: +260 978124 392, groesbeck.parham@ cidrz.org. 
conditional probability model, we estimated that our program prevented 142 cervical cancer deaths (high/low range: 238-96) among the 6572 HIV-infected women screened, or one cervical cancer death prevented per 46 (corresponding range: 28-68) HIV-infected women screened.

Conclusion-Our prevention efforts using setting-appropriate human resources and technology have reduced morbidity and mortality from cervical cancer among HIV-infected women in Zambia. Financial support for implementing cervical cancer prevention programs integrated within HIV/AIDS care programs is warranted. Our prevention model can serve as the implementation platform for future low-cost HPV-based screening methods, and our results may provide the basis for comparison of programmatic effectiveness of future prevention efforts.

\section{Keywords}

cervical cancer; HIV/AIDS; program effectiveness; screening; Zambia

Invasive cervical cancer (ICC) remains the leading cause of cancer-related mortality and morbidity in low-income countries of the developing world [1,2]. Over the past decade, a variety of efforts (including randomized clinical trials) have demonstrated the utility of noncytological prevention methods such as visual inspection with acetic acid (VIA) and human papilloma-virus (HPV)-DNA testing [3,4]. While such efforts have shown the safety, acceptability, feasibility and clinical effectiveness of such 'screen and treat' (linking screening test results to immediate cryotherapy) prevention services for reducing cervical cancer incidence and mortality in the developing world, measuring effectiveness in routine program implementation settings has remained a challenge [3-6].

HIV-infected women are at high risk for developing cervical intraepithelial neoplasia (CIN) and ICC when they are persistently coinfected with oncogenic HPV genotypes, immunosuppressed due to HIV and without access to effective cervical cancer screening services [7-9]. Such circumstances characterize large numbers of HIV-infected women residing in low-income nations, at a time when their life spans are being prolonged due to expanded access to effective antiretroviral therapy and medical care [10,11]. Our previous efforts demon strated an extraordinarily high burden of HPV and cervical disease among HIV-infected women in Zambia [7,12]. This prompted us to launch a cervical cancer prevention program targeting HIV-infected women, with the ultimate goal of expanding to all Zambian women and decreasing cervical cancer-associated morbidity and mortality. Our effort was supported by a partnership between Zambian and US academic (University Teaching Hospital [UTH] in Lusaka; University of Alabama in Birmingham [UAB]), research (Centre for Infectious Disease Research in Zambia [CIDRZ]) and government (Zambian Ministry of Health) partner institutions. Prevention efforts have been scaled up rapidly over 3 years with substantial assistance from the US President's Emergency Plan for AIDS Relief (PEPFAR) and have cumulatively provided screening services to over 52,000 women as of 1st October 2010 [13-15]. In this Research Article, we systematisystematically analyze these implementation efforts by measurement and modeling of the real-world program effectiveness through measurement of outcomes of program uptake, screening test efficacy and treatment effectiveness, and then extrapolating its impact on reductions in mortality. 


\section{Methods}

The program implementation details have been published previously [13-15]. Brie y, cervical can-y, cervical cancer prevention services are located within government-operated public health clinics that provide much of the primary care for a large majority of Zambians. PEPFAR-funded, UAB-CIDRZ-supported HIV care and treatment services in Zambia are tightly integrated into 53 of these government-operated facilities [10]. Since the cervical cancer program's inception in January 2006, 18 Zambian nurses have been trained in conducting cervical cancer screening examinations by VIA. We added digital imaging of the cervix through a portable commercial brand digital camera (digital cervicography) as a routine adjunct to VIA to assist with patient and provider education and quality control [14]. Nurses operate independently in rooms co-located within 15 public health clinics (11 urban and four rural) operated by the Zambian Ministry of Health. Women who test VIA positive and have eligible lesions are offered cryotherapy immediately following screening [13,15]. When necessary, nurses consult local gynecologists by distance consultation using electronic transfer of digital photographs of the screened cervix to help make the appropriate cryotherapy or referral decisions [14]. Those with lesions requiring histological evaluation are referred to a Gynecologic Cancer Prevention Unit at UTH [15]. All tissue specimens were interpreted in Zambia by a board-certified Zambian senior pathologist (VM) in the Department of Pathology at UTH. Patients diagnosed with ICC are treated at UTH (surgery) and the Cancer Diseases Hospital (radiation/chemotherapy), depending on the stage of disease and medical condition of the patient. Basic sociodemographic and clinical data are transferred from paper-based clinic records to a centralized electronic medical records database. Follow-up visits for those undergoing local ablative (cryotherapy) or excisional therapy using the loop electrosurgical excision procedure (LEEP), in a 'see and LEEP' format (i.e., electrosurgical excision guided by digital camera photographic images and without preceding punch biopsies), are encouraged at 6 weeks (assess complications, discuss pathology report) and 6 and 12 months (for rescreening by digital cervicography-aided VIA to detect persistent and recurrent lesions). Annual screening is recommended for all, including those who initially screened negative, for continued surveillance of cervical disease status. Following all treatments, women are specifically asked to avoid sexual intercourse for 6 weeks and are provided condoms to reduce the risk of infection (to a woman if she is HIV-uninfected or to her and her partner if the woman is HIV-infected) in case intercourse cannot be avoided.

In this analysis, we measured the clinical and programmatic outcomes of women (all women and HIV-infected women) enrolled in the cervical cancer prevention program. Their demographic characteristics are summarized using means and standard deviations or medians and interquartile ranges (for continuous variables) and frequency counts and percentages (for categorical variables). We used this information and other published literature to develop a conditional probability model to estimate program effectiveness, as measured by the total number of cervical cancer deaths prevented among the screened population of HIV-infected women as a result of these early detection and treatment interventions. We performed sensitivity (uncertainty) analyses, estimating potential high and low effects, using varying assumptions from the published literature of three major attributes 
that determine screening program effectiveness: first, performance of the screening test, as measured by the predictive value of a positive screening test (digital cervicography-aided VIA) [16-18]; second, progression rate [8,19-23] of the disease as informed by natural history studies; and third, cure rates [24-26] for various treatment modalities including cryotherapy, local excision, surgery and chemo/radiation therapy, for all grades of preinvasive neoplasia (CIN 1-3) and stages of cancer (1A-IV).

For neoplasia grades and cancer stages; the number of observed cases was first multiplied by the positive predictive value estimate of the screening test to get the estimate of the 'true' disease cases that were prevented. This was then multiplied by the progression rate to arrive at the estimated number of cancer cases that would have occurred had the disease gone untreated. The estimated number of cancer cases was then multiplied by the cure rates to determine the number of cancer deaths prevented, which was further used to estimate the number of women needed to be screened to prevent one death due to cervical cancer in the population of HIV-infected women screened. Sensitivity analyses were performed to examine the impact of various combinations $(n=27)$ of high, low and average estimates of predictive values of a positive screening test, disease progression rates and cure rates by various treatment modalities on our estimate. The global literature used for assumptions in our sensitivity range estimates were from limited studies among HIV-infected women or from estimates from the general population when no such data are available from studies in HIV-infected women (e.g., progression/cure rates for various stages of ICC). Analyses were performed using SAS ${ }^{\mathrm{TM}}$ v9.13 (SAS Institute, Cary, NC, USA) and Microsoft Excel ${ }^{\mathrm{TM}}$ (Microsoft Corporation, Redmond, WA, USA).

\section{Results}

\section{Clinical outcomes \& uptake indicators}

From January 2006 through December 2008, we screened 21,010 women for cervical cancer, of whom $6572(31.3 \%)$ were HIV infected, 7374 (35.1\%) were HIV seronegative while HIV serostatus was unknown in 7064 (33.6\%) women (Figure 1). Selected relevant socio demographic characteristics of the HIV-infected population were mean age: 34.2 years $(\mathrm{SD} \pm 8.0)$; mean age at first sex: 17.6 years $(\mathrm{SD} \pm 2.6) ; 49 \%$ with primary school education or below; $68 \%$ with income less than 500,000 kwacha/month ( US\$125/month or US\$ 4/ day); and 53\% married and cohabitating with their husbands. Median $\mathrm{CD} 4^{+} \mathrm{T}$-cell count

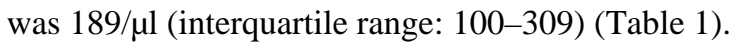

More than half (54\% [3523/6572]) of the HIV-infected women who underwent screening had evidence of a visually apparent lesion suggestive of precancer ('VIA positive'), of whom about three-fifths (59\% [2061/3523]) had visual lesions eligible for cryotherapy. Of these women eligible for ablative treatment by cryotherapy, $78 \%$ (1603/2061) actually underwent treatment, two-thirds (1079/1603) on the same day as screening and the rest (524/1603) delayed treatment, for which the median time of delay was 11 days (interquartile range: $4-56.5$ days). The remaining $22 \%$ (458/2061) who were eligible for cryotherapy never returned for treatment. 75\% (1095/1462) of HIV-infected women referred for histologic evaluation were compliant with recommendations to attend the Gynecologic Cancer Prevention Clinic at the UTH, while 25\% (367/1462) were 'no shows'. Pathology 
reports were available for 65\% (715/1095) of patients that underwent histologic evaluation, while the rest (35\% [380/1095]) were not available (lost before data entry, lost during record keeping or had a loss of specimen during processing). The pathology results included benign abnormalities in 151 out of 715 (21\%), CIN 1 in 214 out of 715 (30\%), CIN 2/3 in 235 out of $715(33 \%)$ and ICC in 115 out of $715(16.1 \%)$. Early stage (1A-1B) cancers made up over two third (69\% [79/115]) of ICCs (Figure 2).

Indicators of program uptake, follow-up and treatment outcomes revealed that over a third (35\%) of HIV-infected women underwent either ablative (cryotherapy) or excisional (LEEP) treatment (Figure 3). However, less than $20 \%$ of women ever returned for their recommended follow-up visit (6 months for those undergoing cryotherapy or LEEP) and 1 year (for VIA-negative women) (Figure 4). Visual lesions were 'positive' (suggestive of recurrent or persistent lesions) in about a third (34\% [89/260]) of the HIV-infected women treated with cryotherapy who returned for follow-up at 6 months. Among these women, available histological evaluation (by LEEP excision) revealed CIN 2+ lesions in $34 \%$ (18/53). Among HIV-infected women undergoing LEEP who returned for a 6-month followup, visual lesions were 'positive' in over two thirds (68\% [64/94]). Among these, CIN 2+ on histology was present in 40\% (19/47) women who underwent repeat excision (Figure 5).

\section{Modeling program effectiveness}

Using the average estimates from our data of the three aforementioned attributes that would impact program effectiveness, we estimated that 142 cancer deaths were prevented by screening 6572 HIV-infected women, or one cancer death averted for every $46 \mathrm{HIV}$-infected woman screened (Table $2 \&$ Figure 6). Using the 27 permutation combinations of low, average and high estimates of the positive predictive value, progression rates and cure rates (as estimated from our global literature review), we estimate that our program prevented between 238 (high estimate for high predictive value, high progression rate and high cure rate) and 96 (low estimate for low predictive value, low progression rate and low cure rate) cervical cancer deaths in $6572 \mathrm{HIV}$-infected women, a range of one cervical cancer death prevented for every 28-68 HIV-infected women screened, respectively (Figure 6).

\section{Discussion}

Zambia has the second highest incidence rates of ICC in the world (52.8 per 100,000 women) [27] and its HIV prevalence is also among the world's highest (over 20\% in reproductive age group adults in 2008) [28,29]. The high background cervical disease prevalence among HIV-infected women (as demonstrated by the high screen positive rate of $53 \%$, and histologically confirmed prevalence of $63 \% \mathrm{CIN}$ [all grades], $32 \% \mathrm{CIN} 2 / 3$, and $16 \%$ ICC among those referred; much higher than other studies in similar populations [30-32]) is juxtaposed with the severe lack of availability and access to screening. In this low-income African nation in which less than $5 \%$ of the population had ever been screened for cervical cancer [2], knowledge of cervical cancer is fraught with myths and misunderstandings [33]. Nevertheless, more than 21,000 women voluntarily accessed our nurse-led 'screen and treat' cervical cancer prevention services during the first 3 years of program operations; more than 6,500 of these women were HIV-infected. 
The Zambian Cervical Cancer Prevention Program is the first large-scale public sector cervical cancer prevention intervention effort within PEPFAR-supported programs. Although initiated as an intervention targeting HIV infected women, cervical cancer prevention is now part of routine health service delivery for Zambian women, regardless of HIV serostatus as a result of our program. The results of the effectiveness model points to the major life-saving potential of an 'once-in-a-lifetime' screening and treatment intervention for cervical cancer that was integrated with an ongoing HIV/AIDS care and treatment program in one of the most resource-limited settings in sub-Saharan Africa. It also points to a very high 'intervention-to-impact' ratio, especially on the background of a very high prevalence of cervical neoplastic disease and HIV prevalence in this population. The rate of being able to prevent one cancer death for every 46 HIV-infected women screened is therefore re ective of the substantial impact that a screening program can deliver in previously unscreened populations with high prevalence rates.

Our conditional probability model, while comprehensive, must be viewed through the prism of the substantial limitations that are inherent in parameter inputs and assumptions, especially when very limited rigorously collected primary data exists from similar settings and populations [34]. Obvious limitations are the validity of the assumptions used for predictive values, progression rates and cure rates. We relied on the few studies reporting performance of digital cervicography-aided VIA [16-18], although none were reporting specifically among HIV-infected women. Natural history studies of cervical neoplasia in HIV-infected women are few in number [19,21,35-37], and those that do exist differ in terms of median age and length of patient follow-up, histologic confirmation, degree of HIV-related immunosuppression and ARV status, all of which can impact results. Likewise, to the best of our knowledge there is little in the literature describing 5-year survival rates following radiotherapy or surgery for cervical cancer in HIV-infected patients [25,26]. Indeed, progression rates between various stages of cancer can be different, and this difference is modulated by the timing of their detection and ultimate treatment [38]. However, the lack of any data on this 'down-staging' effect made it impractical to include it in the model. We thus gauged the impact of the wide variation of estimates of predictive values, progression and cure rates by computing values of 27 permutation and combinations of low, average and high estimates of these three attributes for our model. In absence of hard data to inform these assumptions, this ana lysis only provides us the basis for comparison for future program effectiveness studies that may incorporate new data on natural history and treatment of HPV/cervical disease in HIV-infected women, as well as refined measures of programmatic inputs and outcomes (cost-effectiveness ana lysis of this effort is currently underway). Finally, we did not account for the impact of follow-up rates on treatment effectiveness in this model. Participation and follow-up rates require a whole series of assumptions, most of which are dependent on local factors such as the sociocultural context, women's economic capacity and preferences, and success of outreach efforts. In any case, those are not linked to parameters of natural history or clinical treatment that were derived from our program outcomes or extrapolated from similar studies from other HIV-infected populations to develop this model. Future research efforts to refine our estimates should undertake variability in follow-up rates and other extraneous factors that might affect program effectiveness. 
Outcomes from intensively followed cohorts of HIV-infected women from resource-rich settings [36,39-41] clearly demonstrate that death from ICC can be prevented if the disease is detected early, appropriate treatment is accessible, timely pathologic ana lysis is available, and mechanisms for effective follow-up are in place. While the outcomes of our program clearly demonstrate that we can address some if not all of these pre-requisites through integrated programming, substantial challenges still remain. Our treatment effectiveness indicators are still low, and are re ective of the substantial HPV disease burden and cervical disease milieu [7,12], as well as the limitations in treatment methods and provider efficiency. We face serious challenges associated with adherence to follow-up recommendations and an under-capacitated laboratory infrastructure. These circumstances are endemic to the environment in which we operate and represent major limitations to the success of our prevention efforts. However, with sustained support through a spectrum of funding agencies we are addressing most of these issues head-on with an overarching goal for providing the best quality of care for all Zambian women accessing our program.

Each year over half a million women will continue to be newly diagnosed with ICC globally, and in the same period over a quarter of a million will die [1]. The vast majority of these women reside in low-income nations such as Zambia, where less than 5\% of women are ever screened in their lifetime for cervical cancer [2]. HPV-based primary (i.e., vaccine) and secondary (i.e., HPV-based screening) approaches have the potential to significantly reduce the global cervical cancer burden over the following decades. However, successful implementation of future molecular HPV-based prevention methods can benefit by the availability of suitable clinical platforms such as the one established through our program. Innovative approaches to disease prevention must avoid placing additional burdens on health systems that are already overburdened. One such strategy is to use the availability, momentum and capacity building efforts resulting from vertical HIV/AIDS care and treatment programs to implement other disease-specific initiatives such as cervical cancer prevention [42]. Our program provides the much-needed evidence about the usefulness of such integrative approaches. By using a setting-appropriate protocol for cervical cancer prevention, for example, nurse-led visual screening and same-visit cryotherapy for eligible women, and linkage to a tertiary care centre for evaluation, our program has not just saved lives but has also established a new paradigm for routine prevention interventions in resource-constrained environments.

\section{Acknowledgments}

Financial \& competing interests disclosure

This work was supported in part by US Centers for Disease Control and Prevention through the President's Emergency Plan for AIDS Relief (PEPFAR) and the Fogarty International Center and National Cancer Institute of the US National Institutes of Health. The funders had no role in the study design, data collection and analysis, decision to publish or preparation of this manuscript.The authors have no other relevant affiliations or financial involvement with any organization or entity with a financial interest in or financial conflict with the subject matter or materials discussed in the manuscript apart from those disclosed.

No writing assistance was utilized in the production of this manuscript. 


\section{Bibliography}

1. Parkin DM, Bray F, Ferlay J, Pisani P. Global cancer statistics, 2002. CA Cancer J. Clin. 2005; 55(2):74-108. [PubMed: 15761078]

2. Gakidou E, Nordhagen S, Obermeyer Z. Coverage of cervical cancer screening in 57 countries: low average levels and large inequalities. PLoS Med. 2008; 5(6):e132. [PubMed: 18563963]

3. Sankaranarayanan R, Esmy PO, Rajkumar R, et al. Effect of visual screening on cervical cancer incidence and mortality in Tamil Nadu, India: a cluster-randomised trial. Lancet. 2007; 370(9585): 398-406. [PubMed: 17679017]

4. Denny L, Kuhn L, De Souza M, Pollack AE, Dupree W, Wright TC Jr. Screen-and-treat approaches for cervical cancer prevention in low-resource settings: a randomized controlled trial. JAMA. 2005; 294(17):2173-2181. [PubMed: 16264158]

5. Blumenthal PD, Gaffikin L, Deganus S, Lewis R, Emerson M, Adadevoh S. Cervical cancer prevention: safety, acceptability, and feasibility of a single-visit approach in Accra, Ghana. Am. J. Obstet. Gynecol. 2007; 196(4):407 e401-408. discussion 407 e408-409. [PubMed: 17403438]

6. Gaffikin L, Blumenthal PD, Emerson M, Limpaphayom K. Safety, acceptability, and feasibility of a single-visit approach to cervical-cancer prevention in rural Thailand: a demonstration project. Lancet. 2003; 361(9360):814-820. [PubMed: 12642047]

7. Parham GP, Sahasrabuddhe VV, Mwanahamuntu MH, et al. Prevalence and predictors of squamous intraepithelial lesions of the cervix in HIV-infected women in Lusaka, Zambia. Gynecol. Oncol. 2006; 103(3):1017-1022. [PubMed: 16875716]

8. Denny L, Boa R, Williamson AL, et al. Human papillomavirus infection and cervical disease in human immunodeficiency virus-1-infected women. Obstet. Gynecol. 2008; 111(6):1380-1387. [PubMed: 18515522]

9. Clifford GM, Polesel J, Rickenbach M, et al. Cancer risk in the Swiss HIV Cohort Study: associations with immunodeficiency, smoking, and highly active antiretroviral therapy. J. Natl Cancer Inst. 2005; 97(6):425-432. [PubMed: 15770006]

10. Stringer JS, Zulu I, Levy J, et al. Rapid scale-up of antiretroviral therapy at primary care sites in Zambia: feasibility and early outcomes. JAMA. 2006; 296(7):782-793. [PubMed: 16905784]

11. Mermin J, Were W, Ekwaru JP, et al. Mortality in HIV-infected Ugandan adults receiving antiretroviral treatment and survival of their HIV-uninfected children: a prospective cohort study. Lancet. 2008; 371(9614):752-759. [PubMed: 18313504]

12. Sahasrabuddhe VV, Mwanahamuntu MH, Vermund SH, et al. Prevalence and distribution of HPV genotypes among HIV-infected women in Zambia. Br. J. Cancer. 2007; 96(9):1480-1483. [PubMed: 17437020]

13. Mwanahamuntu MH, Sahasrabuddhe VV, Pfaendler KS, et al. Implementation of 'see-and-treat' cervical cancer prevention services linked to HIV care in Zambia. AIDS. 2009; 23(6):N1-N5. [PubMed: 19279439]

14. Parham GP, Mwanahamuntu MH, Pfaendler KS, et al. eC3 - a modern telecommunications matrix for cervical cancer prevention in Zambia. J. Low. Genit. Tract Dis. 2010; 14(3):167-173. [PubMed: 20592550]

15. Pfaendler KS, Mwanahamuntu MH, Sahasrabuddhe VV, Mudenda V, Stringer JS, Parham GP. Management of cryotherapy-ineligible women in a "screen-and-treat" cervical cancer prevention program targeting HIV-infected women in Zambia: lessons from the field. Gynecol. Oncol. 2008; 110(3):402-407. [PubMed: 18556050]

16. Bomfim-Hyppolito S, Franco ES, Franco RG, de Albuquerque CM, Nunes GC. Cervicography as an adjunctive test to visual inspection with acetic acid in cervical cancer detection screening. Int. J. Gynaecol. Obstet. 2006; 92(1):58-63. [PubMed: 16269147]

17. Cremer ML, Peralta EI, Dheming SG, et al. Digital assessment of the reproductive tract versus colposcopy for directing biopsies in women with abnormal Pap smears. J. Low Genit. Tract Dis. 2010; 14(1):5-10. [PubMed: 20040830]

18. Cremer M, Jamshidi RM, Muderspach L, Tsao-Wei D, Felix JC, Blumenthal PD. Digital camera assessment for detection of cervical intraepithelial neoplasia in rural El Salvador. Int. J. Gynaecol. Obstet. 2005; 91(1):42-46. [PubMed: 16043183] 
19. Massad LS, Evans CT, Minkoff H, et al. Natural history of grade 1 cervical intraepithelial neoplasia in women with human immunodeficiency virus. Obstet. Gynecol. 2004; 104(5 Pt 1): 1077-1085. [PubMed: 15516404]

20. Nappi L, Carriero C, Bettocchi S, Herrero J, Vimercati A, Putignano G. Cervical squamous intraepithelial lesions of low-grade in HIV-infected women: recurrence, persistence, and progression, in treated and untreated women. Eur. J. Obstet. Gynecol. Reprod. Biol. 2005; 121(2): 226-232. [PubMed: 16054967]

21. Delmas MC, Larsen C, van Benthem B, et al. European Study Group on Natural History of HIV Infection in Women. Cervical squamous intraepithelial lesions in HIV-infected women: prevalence, incidence and regression. AIDS. 2000; 14(12):1775-1784. [PubMed: 10985315]

22. Robinson WR, Luck MB, Kendall MA, Darragh TM. The predictive value of cytologic testing in women with the human immunodeficiency virus who have low-grade squamous cervical lesions: a substudy of a randomized, phase III chemoprevention trial. Am. J. Obstet. Gynecol. 2003; 188(4): 896-900. [PubMed: 12712082]

23. Robinson WR, Hamilton CA, Michaels SH, Kissinger P. Effect of excisional therapy and highly active antiretroviral therapy on cervical intraepithelial neoplasia in women infected with human immunodeficiency virus. Am. J. Obstet. Gynecol. 2001; 184(4):538-543. [PubMed: 11262450]

24. Stein MD, Cunningham WE, Nakazono T, et al. Screening for cervical cancer in HIV-infected women receiving care in the United States. J. Acquir. Immune Defic. Syndr. 2001; 27(5):463-466. [PubMed: 11511823]

25. Maiman M, Fruchter RG, Guy L, Cuthill S, Levine P, Serur E. Human immunodeficiency virus infection and invasive cervical carcinoma. Cancer. 1993; 71(2):402-406. [PubMed: 8093678]

26. Moodley M. Radical hysterectomy for cervical cancer amongst women infected with the human immunodeficiency virus. Int. J. Gynecol. Cancer. 2007; 17(6):1264-1265. [PubMed: 17433062]

27. Ferlay J, Shin HR, Bray F, Forman D, Mathers C, Parkin DM. Estimates of worldwide burden of cancer in 2008: GLOBOCAN 2008. Int. J. Cancer. 2010 Epub ahead of print.

28. Hallett TB, Stover J, Mishra V, Ghys PD, Gregson S, Boerma T. Estimates of HIV incidence from household-based prevalence surveys. AIDS. 2010; 24(1):147-152. [PubMed: 19915447]

29. Stringer EM, Chintu NT, Levy JW, et al. Declining HIV prevalence among young pregnant women in Lusaka, Zambia. Bull World Health Organ. 2008; 86(9):697-702. [PubMed: 18797645]

30. Akinwuntan AL, Adesina OA, Okolo CA, et al. Correlation of cervical cytology and visual inspection with acetic acid in HIV-positive women. J. Obstet. Gynaecol. 2008; 28(6):638-641. [PubMed: 19003664]

31. Singh DK, Anastos K, Hoover DR, et al. Human papillomavirus infection and cervical cytology in HIV-infected and HIV-uninfected Rwandan women. J. Infect. Dis. 2009; 199(12):1851-1861. [PubMed: 19435429]

32. Sahasrabuddhe VV, Bhosale RA, Joshi SN, et al. Prevalence and predictors of colposcopichistopathologically confirmed cervical intraepithelial neoplasia in HIV-infected women in India. PLoS One. 2010; 5(1):e8634. [PubMed: 20072610]

33. Chirwa S, Mwanahamuntu M, Kapambwe S, et al. Myths and misconceptions about cervical cancer among Zambian women: rapid assessment by peer educators. Glob. Health Promot. 2010; 17(2 Suppl.):47-50. [PubMed: 20595342]

34. Louie KS, de Sanjose S, Mayaud P. Epidemiology and prevention of human papillomavirus and cervical cancer in sub-Saharan Africa: a comprehensive review. Trop. Med. Int. Health. 2009; 14(10):1287-1302. [PubMed: 19772550]

35. Strickler HD, Burk RD, Fazzari M, et al. Natural history and possible reactivation of human papillomavirus in human immunodeficiency virus-positive women. J. Natl Cancer Inst. 2005; 97(8):577-586. [PubMed: 15840880]

36. Heard I, Tassie JM, Schmitz V, Mandelbrot L, Kazatchkine MD, Orth G. Increased risk of cervical disease among human immunodeficiency virus-infected women with severe immunosuppression and high human papillomavirus load(1). Obstet. Gynecol. 2000; 96(3):403-409. [PubMed: 10960634] 
37. Heard I, Schmitz V, Costagliola D, Orth G, Kazatchkine MD. Early regression of cervical lesions in HIV-seropositive women receiving highly active antiretroviral therapy. AIDS. 1998; 12(12): 1459-1464. [PubMed: 9727566]

38. Richart RM. Screening. The next century. Cancer. 1995; 76(10 Suppl.):1919-1927. [PubMed: 8634983]

39. Massad LS, Seaberg EC, Watts DH, et al. Long-term incidence of cervical cancer in women with human immunodeficiency virus. Cancer. 2009; 115(3):524-530. [PubMed: 19127538]

40. Russomano F, Reis A, Camargo MJ, Grinsztejn B, Tristao MA. Recurrence of cervical intraepithelial neoplasia grades 2 or 3 in HIV-infected women treated by large loop excision of the transformation zone (LLETZ). Sao Paulo Med. J. 2008; 126(1):17-22. [PubMed: 18425282]

41. Grulich AE, van Leeuwen MT, Falster MO, Vajdic CM. Incidence of cancers in people with HIV/ AIDS compared with immunosuppressed transplant recipients: a meta-ana lysis. Lancet. 2007; 370(9581):59-67. [PubMed: 17617273]

42. Denny CC, Emanuel EJ. US health aid beyond PEPFAR: the Mother \& Child Campaign. JAMA. 2008; 300(17):2048-2051. [PubMed: 18984893] 


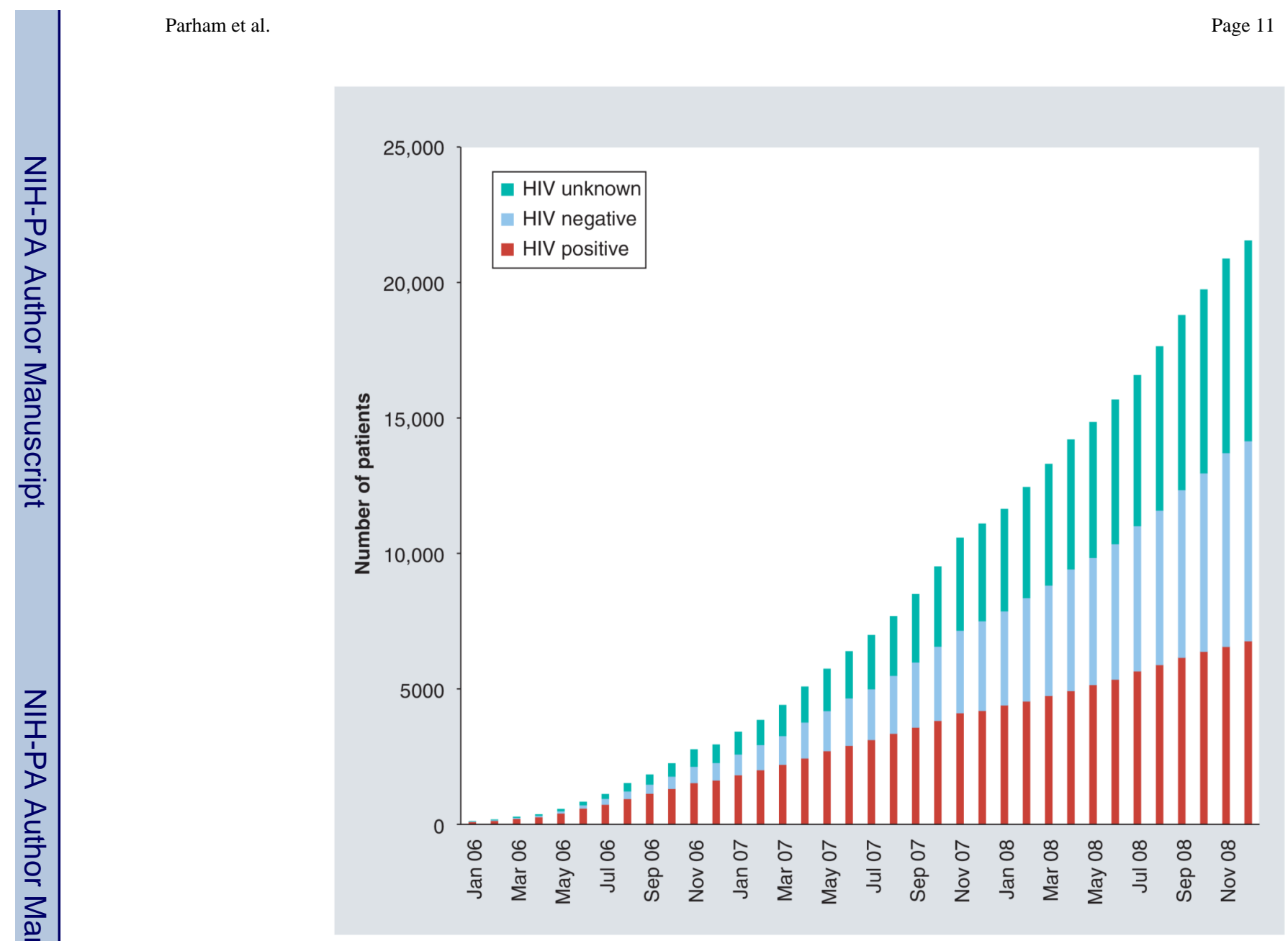

Figure 1. Cumulative patient enrollment from January 2006 to December 2008, Zambian Cervical Cancer Prevention Program $(n=21,010)$ 


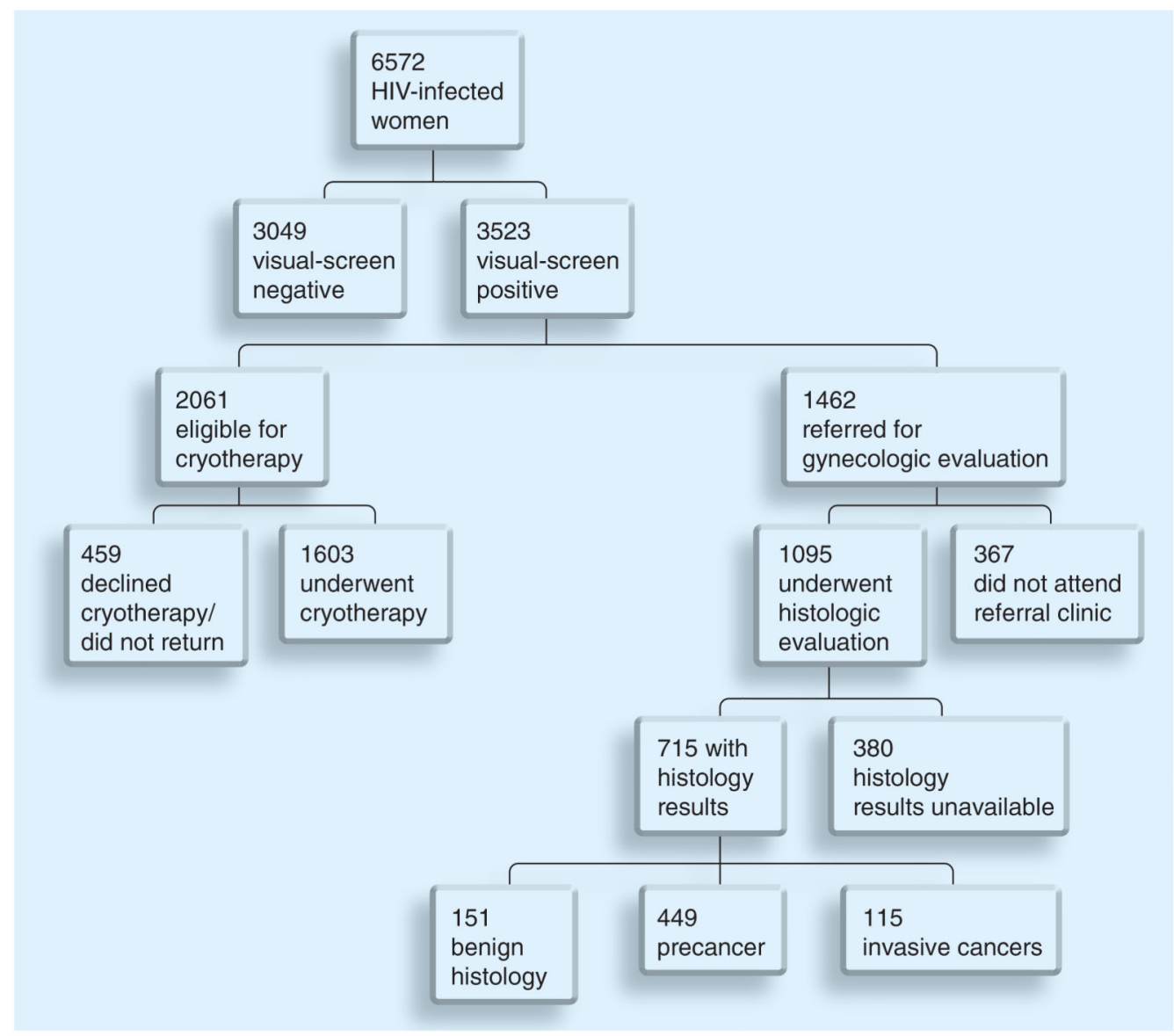

Figure 2. Patient flow chart for clinical screening and care in the Zambian Cervical Cancer Prevention Program 


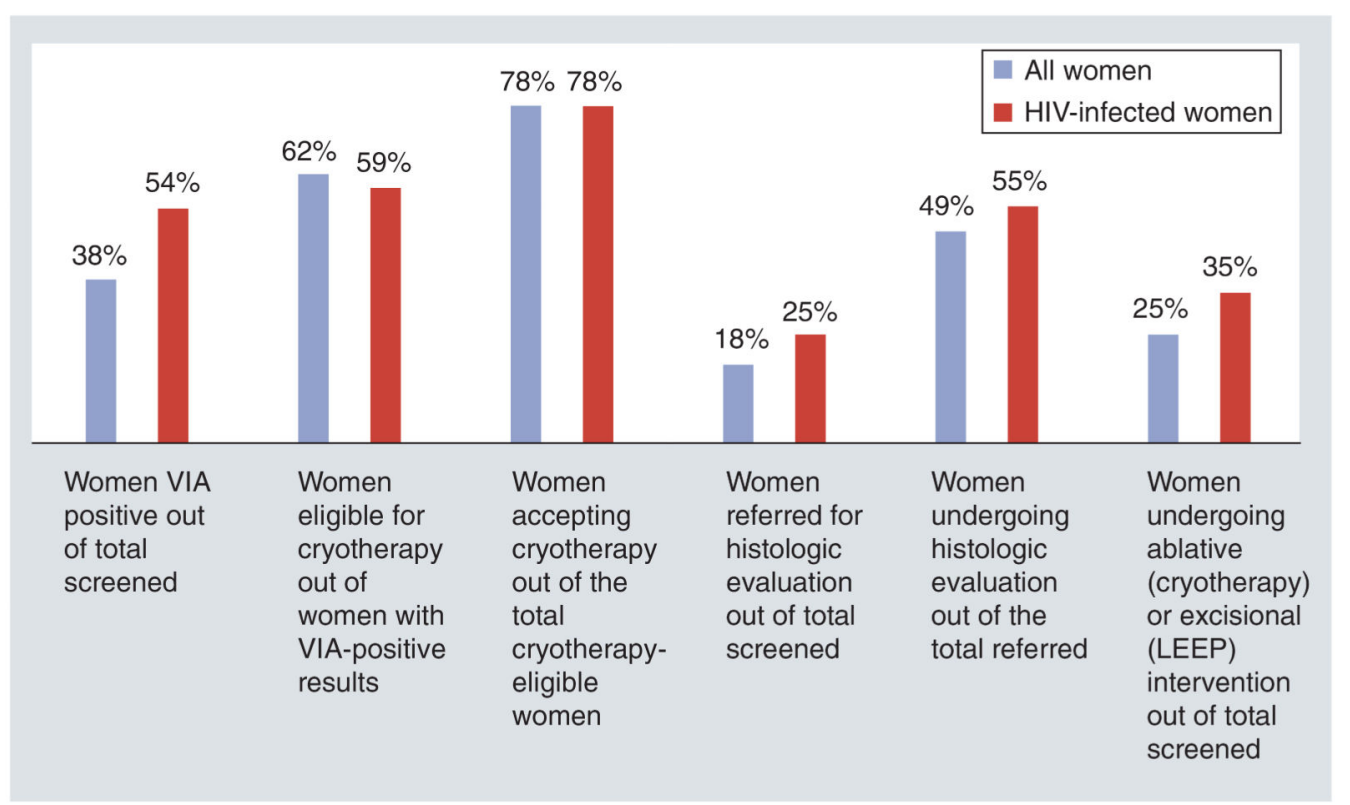

Figure 3. Indicators of participation in screening program (all women and HIV-infected women) LEEP: Loop electrosurgical excision procedure; VIA: Visual inspection with dilute acetic acid. 


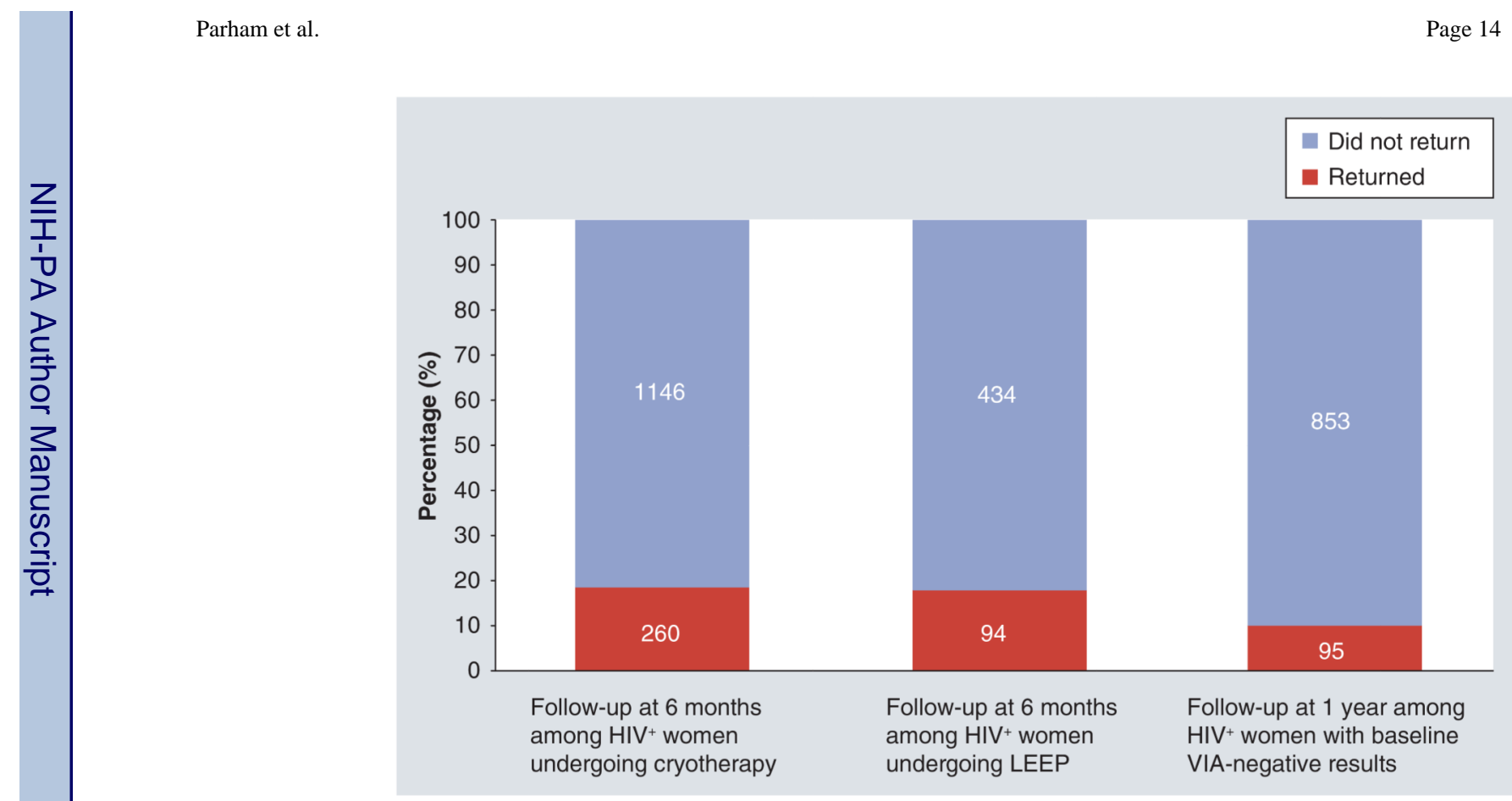

Figure 4. Rates of follow-up among HIV-infected women at the recommended 6 month or 1 year visits effectiveness

LEEP: Loop electrosurgical excision procedure; VIA: Visual inspection with dilute acetic acid. 


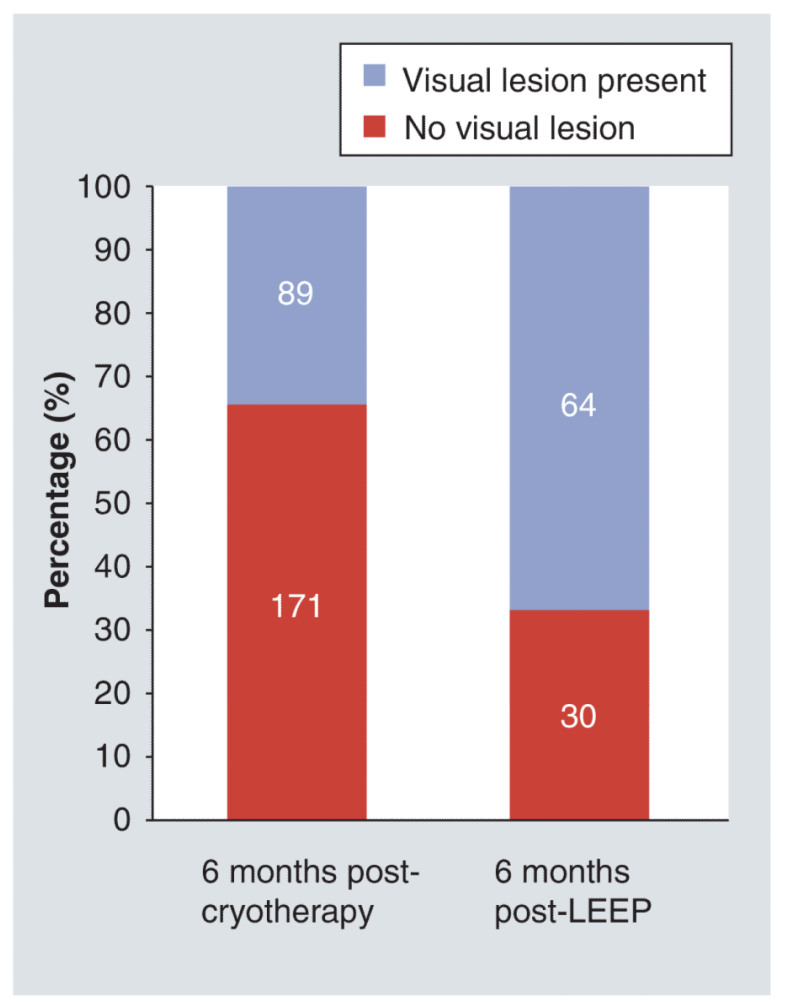

Figure 5. Indicators of treatment effectiveness among HIV-infected women who return for follow-up at 6 months post-cryotherapy or post-LEEP procedures LEEP: Loop electrosurgical excision procedure. 


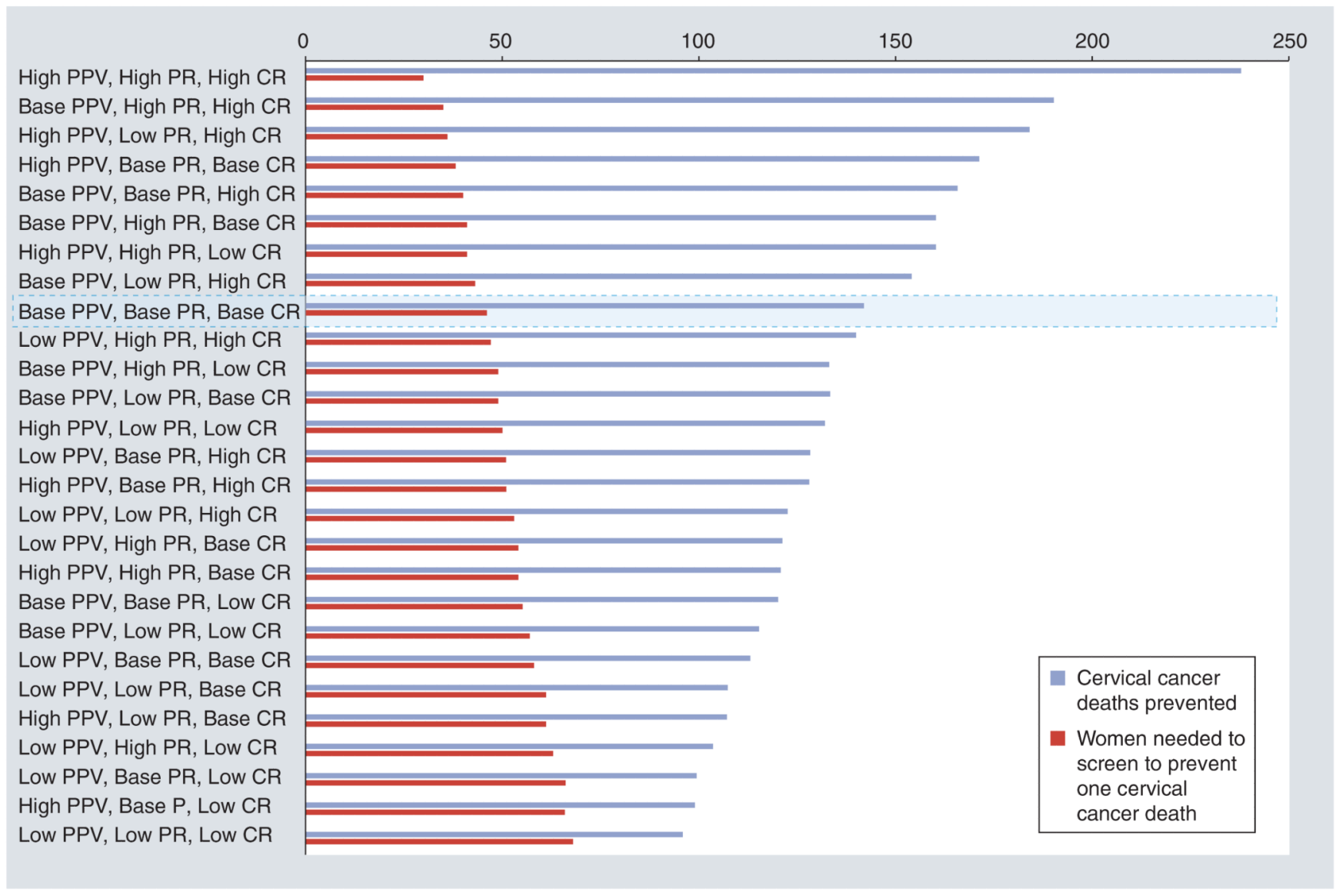

Figure 6. Tornado graph showing the uncertainty ana lysis for the conditional probability model to measure program effectiveness of the Zambian Cervical Cancer Prevention Program

The Tornado graph shows trend in the estimates of: total number of cervical cancer deaths prevented (blue bars); and total number of women needed to be screened to prevent one cervical cancer death (red bars). The graph shows 27 permutation/combinations of the three scenarios: 'base' case, 'high' estimates and 'low' estimates of the three major attributes used in the conditional probability model; (i) measure of test performance (as measured by the PPV), (ii) disease PR and (iii) CR for various treatment modalities. The estimates of the total cancer deaths prevented estimated under each permutation/combination are arranged in a declining order on the left axis. The corresponding number of women needed to prevent one cancer death under that scenario, plotted along the same axis, is shown in ascending order. The outcomes of the model in the base case scenario are highlighted in the Tornado graph. CR: Cure rate; PPV: Positive predictive value; PR: Progression rate. 
Table 1

Sociodemographic and clinical characteristics for women, stratified by HIV status, attending clinics in I the Zambian Cervical Cancer Prevention Program from 2006 to 2008.

\begin{tabular}{|c|c|c|c|c|}
\hline & $\begin{array}{l}\text { Total screened } \\
\left(\mathrm{n}=\mathbf{2 1 , 0 1 0 ^ { \dagger }}\right)\end{array}$ & $\begin{array}{l}\text { HIV seropositive } \\
(\mathbf{n}=6572)\end{array}$ & $\begin{array}{l}\text { HIV seronegative } \\
(\mathrm{n}=\mathbf{7 2 3 8})\end{array}$ & p-value \\
\hline Age (years), mean (SD) & $33.1(9.8)$ & $34.2(8.0)$ & $31.1(9.5)$ & $<0.01$ \\
\hline Age (years), median (IQR) & $31(12)$ & $33(11)$ & $29(12)$ & $<0.01$ \\
\hline Education primary school and below, $\mathrm{N}(\%)$ & $10,080(48 \%)$ & $3287(49 \%)$ & $3010(42 \%)$ & $<0.01$ \\
\hline $\begin{array}{l}\text { Income }<500,000 \text { Zambian Kwacha per } \\
\text { month }(\sim \mathrm{US} \$ 125), \mathrm{N}(\%)\end{array}$ & $11,924(63 \%)$ & $4108(68 \%)$ & $3599(56 \%)$ & $<0.01$ \\
\hline Married, cohabiting husband, $\mathrm{N}(\%)$ & $13,677(65 \%)$ & $3514(53 \%)$ & $5253(72 \%)$ & $<0.01$ \\
\hline Age at menarche (years), mean (SD) & $14.7(1.8)$ & $14.7(1.8)$ & $14.7(1.8)$ & 0.06 \\
\hline Age at first sex (years), mean (SD) & $17.8(2.7)$ & $17.6(2.6)$ & $18.2(2.9)$ & $<0.01$ \\
\hline $\begin{array}{l}\text { Time between menarche and age at first sex } \\
\text { (years), mean (SD) }\end{array}$ & $3.2(2.7)$ & $3.0(2.5)$ & $3.6(2.9)$ & $<0.01$ \\
\hline Time sexually active (years), mean (SD) & $15.3(9.9)$ & $16.6(8.2)$ & $12.8(9.5)$ & $<0.01$ \\
\hline $\begin{array}{l}\text { Number of lifetime sexual partners, } \\
\text { median (IQR) }\end{array}$ & $2(2)$ & $3(3)$ & $2(2)$ & $<0.01$ \\
\hline Number of pregnancies, median (IQR) & $3(3)$ & $3(3)$ & $3(3)$ & $<0.01$ \\
\hline Number of live births, median (IQR) & $2(3)$ & $3(3)$ & $2(3)$ & $<0.01$ \\
\hline $\begin{array}{l}\text { Never used a condom with regular partner, } \\
\mathrm{N}(\%)\end{array}$ & $12,063(61 \%)$ & $2667(44 \%)$ & $4289(63 \%)$ & $<0.01$ \\
\hline Ever had a Pap smear, N (\%) & $571(3 \%)$ & $173(3 \%)$ & $220(3 \%)$ & 0.12 \\
\hline History of bleeding during coitus, $\mathrm{N}(\%)$ & $2043(10 \%)$ & $741(12 \%)$ & $633(9 \%)$ & $<0.01$ \\
\hline
\end{tabular}

IQR: Interquartile range; SD: Standard deviation.

${ }^{\dagger}$ Total $\mathrm{n}=21,010$ includes 7064 (33.6\%) with HIV status unknown.

F Statistically significant at a of 0.05 for comparison of covariates between HIV-seropositive versus HIV-seronegative women. 
Table 2

Parameters and ranges used for the estimation of cancers prevented to determine program effectiveness in the Zambian Cervical Cancer Prevention Program, 2006-2008.

\begin{tabular}{|c|c|c|c|c|c|c|c|c|}
\hline & \multirow[t]{2}{*}{$\begin{array}{l}\text { Pathology } \\
\text { result }\end{array}$} & \multicolumn{2}{|c|}{$\begin{array}{c}\text { Estimates of predictive } \\
\text { value of positive } \\
\text { screening test }{ }^{\dagger}, \overline{0} \\
\end{array}$} & \multicolumn{2}{|c|}{$\begin{array}{c}\text { Estimates of grade- or } \\
\text { stage-specific disease } \\
\text { progression rates }^{\S} \\
\end{array}$} & \multirow[t]{2}{*}{$\begin{array}{l}\text { Treatment } \\
\text { modality }\end{array}$} & \multicolumn{2}{|c|}{$\begin{array}{c}\text { Estimates of grade- } \\
\text { or stage-specific } \\
\text { disease cure rates }\end{array}$} \\
\hline & & Base case & Low-high range & Base case & $\begin{array}{l}\text { Low-high } \\
\text { range }\end{array}$ & & Base case & $\begin{array}{l}\text { Low-high } \\
\text { range }\end{array}$ \\
\hline VIA positive & & 0.5 & $0.25-0.75$ & 0.06 & $0.056-0.078$ & Cryotherapy & 0.6 & $0.66-0.85$ \\
\hline \multirow[t]{2}{*}{$\mathrm{CIN}$} & CIN 1 & 0.5 & $0.25-0.75$ & 0.06 & $0.056-0.078$ & Local excision & 0.6 & $0.25-0.80$ \\
\hline & CIN 2/3 & 0.5 & $0.25-0.75$ & 0.3 & $0.2-0.4$ & Local excision & 0.6 & $0.25-0.80$ \\
\hline \multirow[t]{3}{*}{ Early-stage cancer } & Stage Ia & 1 & 1 & 1 & 1 & Surgery/XRT & 0.9 & $0.85-1.00$ \\
\hline & Stage Ib & 1 & 1 & 1 & 1 & Surgery/XRT & 0.7 & $0.60-0.90$ \\
\hline & Stage IIa & 1 & 1 & 1 & 1 & Surgery/XRT & 0.7 & $0.65-0.75$ \\
\hline \multirow[t]{5}{*}{ Late-stage cancer } & Stage $11 b$ & 1 & 1 & 1 & 1 & XRT & 0.6 & $0.55-0.65$ \\
\hline & Stage IIIa & 1 & 1 & 1 & 1 & XRT & 0.4 & $0.35-0.45$ \\
\hline & Stage IIIb & 1 & 1 & 1 & 1 & XRT & 0.3 & $0.20-0.40$ \\
\hline & Stage IVa & 1 & 1 & 1 & 1 & XRT & 0.1 & $0.00-0.20$ \\
\hline & Unknown & 1 & 1 & 1 & 1 & XRT & 0.3 & 0.3 \\
\hline
\end{tabular}

CIN: Cervical intraepithelial neoplasia; VIA: Visual inspection with dilute acetic acid; XRT: Radiation therapy.

${ }^{\dagger}$ Adapted from [15-17].

${ }^{\ddagger}$ A positive predictive value (PPV) of a screening test reflects the proportion of women who are truly (confirmed by hlstopathology) positive out of the total women who are screened positive. Thus, it is the appropriate measure of test performance in settings where screening-negative women never get confirmation for true disease (as in this real-world program operation). Note that PPV applies to both VIA and histologic evaluation, such that the average estimate of 0.5 reflects that only $50 \%$ of VIA-positive women were truly diseased, and $50 \%$ of women undergoing histologic evaluation had some CIN lesions. The estimate of 1 for early- and late-stage cancers is used since the combination of histological and clinical staging procedures plus the low inter/intra-observer variability associated with the histologic diagnosis of invasive cancer assumes some stage of cancers is present (hence all women seen for staging have confirmed cancer on histologic evaluation, hence PPV of 1).

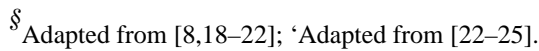

